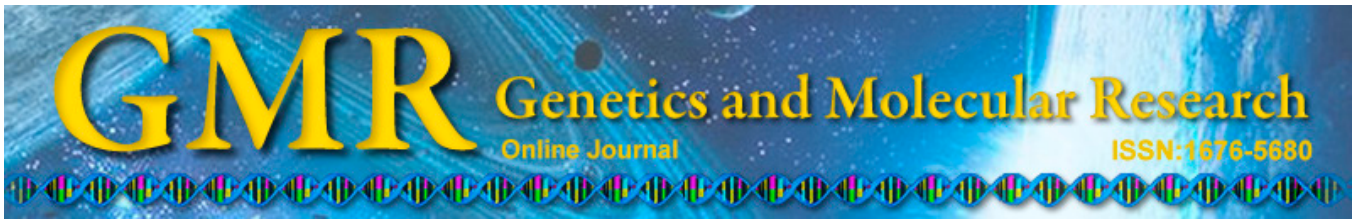

\title{
Effect of $E R C C 1$ polymorphism on the response to chemotherapy and clinical outcome of non-small cell lung cancer
}

\author{
H. Gao ${ }^{1}$, R.C. Ge ${ }^{2}$, H.Y. Liu ${ }^{3}$, Y. Wang ${ }^{4}$ and S. Yan ${ }^{4}$ \\ ${ }^{1}$ Department of General Internal Medicine, \\ Shandong Provincial Jiaotong Hospital, Jinan, China \\ ${ }^{2}$ Department of Regenerative Medicine, \\ Shandong Provincial Jiaotong Hospital, Jinan, China \\ ${ }^{3}$ Affilated Hospital of Tai Shan Medical College, Taian, China \\ ${ }^{4}$ Department of Thoracic Surgery, The Fourth People's Hospital of Jinan, \\ Jinan, China \\ Corresponding author: R.C. Ge \\ E-mail: sdjnsywy1818@163.com
}

Genet. Mol. Res. 13 (4): 8997-9004 (2014)

Received January 11, 2014

Accepted May 21, 2014

Published October 31, 2014

DOI http://dx.doi.org/10.4238/2014.October.31.14

\begin{abstract}
We conducted a cohort study to investigate the role of 3 single-nucleotide polymorphisms of the excision repair crosscomplementation group 1 (ERCC1) gene on the response to chemotherapy and clinical outcomes of non-small cell lung cancer (NSCLC). A total of 163 patients with newly diagnosed and histopathologically confirmed primary NSCLC were examined in our study and were followed up until December 2012. ERCC1 rs11615, rs3212986, and rs2298881 were selected and genotyped. Of the 163 patients, 86 patients showed a complete response and partial response to chemotherapy $(52.76 \%)$, while 91 patients $(55.83 \%)$ died from NSCLC during the follow-up period with a median survival time of 19.3 months (range, 2-60 months). Multivariate regression analysis showed that individuals carrying the rs11615 TT genotype and T allele had a significantly lower response
\end{abstract}


rate to chemotherapy using the rs $11615 \mathrm{CC}$ genotype as the reference. For rs3212986, carriers of the rs3212986 AA genotype and A allele had a significantly lower response rate to chemotherapy when compared with the CC genotype. In the Cox proportional hazards model, patients carrying the rs 11615 TT genotype and T allele and the rs3212986 AA genotype and A allele were significantly associated with increased risk of death from NSCLC. We found that polymorphisms in ERCC1 rs 11615 and rs3212986 were associated with poor response to chemotherapy and shorter survival time of advanced NSCLC.

Key words: Survival time; Single nucleotide polymorphism; Excision repair cross-complementation group 1; Chemotherapy; Non-small cell lung cancer;

\section{INTRODUCTION}

Lung cancer has been the most common cause of death from cancer worldwide for several decades. By 2008, an estimated 1.61 million new lung cancer cases accounted for $12.7 \%$ of all new cancers (IARC, 2012). Non-small cell lung cancer (NSCLC) accounts for approximately $75 \%$ of all cancer-related deaths (Stinchcombe and Socinski, 2008). Most NSCLC patients are diagnosed with metastatic disease and advanced cancer (stage III-IV) and show a 5-year survival rate $<15 \%$ (Molina et al., 2008; Siegel et al., 2012).

Chemotherapy with platinum-based regimens is considered the standard treatment for metastatic and advanced NSCLC (Goffin et al., 2010). However, the response rate to platinum-based chemotherapy is approximately $20 \%$, and drug resistance and lack of effective salvage therapies result in poor prognosis for patients with NSCLC (Tahara et al., 2010). NSCLC patients have different prognoses within the same stage, suggesting that treatments should differ based on individual genetic backgrounds. Previous studies demonstrated that polymorphic changes within several DNA repair genes may be functional and are associated with the risk of various cancers (Krupa et al., 2009; Stanczyk et al., 2011).

Nucleotide excision repair, base excision repair, and double-strand break repair are 3 major pathways for removing DNA damage induced by endogenous and exogenous agents. Excision repair cross-complementation group 1 (ERCC1) is an endonuclease essential in the nucleotide excision repair pathway and plays a role in DNA repair capacity and cancer risk as well as the clinical outcome of cancer (Wang et al., 2005). Recently, several studies suggested that variants in ERCC1 influence the susceptibility to and prognosis of various cancers (Li et al., 2013; Mlak et al., 2013; Yang et al., 2013; De Dosso et al., 2013). Few studies have investigated the role of ERCC1 variants in the clinical outcome in NSCLC (Ozdemir et al., 2013; Mlak et al., 2013). In this prospective study, we analyzed the role of 3 common single-nucleotide polymorphisms (SNPs) in ERCC1 for predicting tumor responses and the clinical outcome of 163 NSCLC patients.

\section{MATERIAL AND METHODS}

\section{Patients}

Between January 2008 and March 2009, 179 patients with stage IV or IIIB/A and who 
received a histopathological diagnosis of advanced NSCLC from The Fourth People's Hospital of Jinan were identified. A total of 163 patients were willing to participate in our study, with a participation rate of $91.06 \%$. All patients received platinum-based chemotherapy as the first-line treatment. Patients who were pregnant or lactating or had cardiopulmonary insufficiency, serious cardiovascular disease, serious infection, or were severely malnourished were excluded. All the patients signed the informed consents, and the ethics approval was obtained from ethics committee of The Fourth People's Hospital of Jinan.

The response to chemotherapy was assessed using the Response Evaluation Criteria in Solid Tumors (Eisenhauer et al., 2009). Patients who showed a complete response (CR) or a partial response (PR) were considered to be responders, while those who presented stable disease (SD) or progressive disease (PD) were defined as non-responders. Overall survival (OS) was calculated as the time from the date of diagnosis until death from any cause or until the date of last follow-up.

\section{Genotyping}

All study participants were asked to provide $5 \mathrm{~mL}$ venous blood, which was stored at $-20^{\circ} \mathrm{C}$ until use, with $0.5 \mathrm{mg} / \mathrm{mL}$ ethylenediaminetetraacetic acid used as an anticoagulant. Genomic DNA was extracted using the TIANamp Blood DNA Kit (Tiangen; Beijing, China) according to manufacturer instructions.

Three SNPs in ERCC1, rs11615, rs3212986, and rs2298881, were selected. The primers used for the rs11615, rs3212986, and rs2298881 loci for polymerase chain reaction (PCR) amplification were designed using the web-based Primer Premier 5.0 software (http://www.premierbiosoft.com/primerdesign/). Genotyping of rs11615, rs3212986, and rs2298881 was conducted on a 384-well plate format on the MassARRAY ${ }^{\circledR}$ Analyzer 4 system [Query 31] (Sequenom $^{\circledR}$; San Diego, CA, USA), which combines PCR and matrix-assisted laser desorption/ionization time-of-flight mass spectrometry technologies. PCR was conducted in a $20-\mu \mathrm{L}$ reaction volume containing 50 ng genomic DNA, $200 \mu \mathrm{M}$ dNTPs, 2.5 U Taq DNA polymerase (Promega Corporation; Madison, WI, USA), and $200 \mu \mathrm{M}$ primers. The PCR reaction started at $94^{\circ} \mathrm{C}$ for 2 min, followed with 35 cycles of $94^{\circ} \mathrm{C}$ for $30 \mathrm{~s}$, the annealing temperature was reduced to $64^{\circ} \mathrm{C}$ for $30 \mathrm{~s}$, and $72^{\circ} \mathrm{C}$ for $1 \mathrm{~min}$. PCR products were verified by $1.0 \%$ agarose gel electrophoresis.

\section{Statistical analysis}

Statistical analysis was conducted using SPSS ${ }^{\circledR}$ version 11.0 (SPSS Inc.; Chicago, IL, USA) for Windows ${ }^{\circledR}$. Continuous variables are reported as the means $\pm S D$, while categorical variables were expressed as the number of subjects (\%). The correlation between $E R C C 1$ polymorphisms and response to chemotherapy was examined using $\chi^{2}$ tests, and their association was assessed using odds ratios (ORs) and their corresponding 95\% confidence intervals (CIs). The Kaplan-Meier method was used to calculate the overall survival time, and the log-rank test was used to determine distributions. Cox proportional hazards regression was used to analyze the prognostic value of the 3 SNPs in ERCC1 for NSCLC, with hazard ratios (HRs) and their 95\%CI. P $<0.05$ was considered to be statistically significant. All tests were 2-sided. 


\section{RESULTS}

\section{Subject characteristics}

Of the 163 patients screened for eligibility, 116 (71.17\%) were male and 47 (28.83\%) were female (Table 1). The average age was $61.6 \pm 9.2$ years. There were 92 current or ever smokers (56.44\%). A total of $105(64.42 \%)$ were at 0-1 Eastern Cooperative Oncology Group performance status, $108(66.26 \%)$ were at stage IV disease, and $93(57.06 \%)$ patients had nonsquamous cell carcinoma.

\begin{tabular}{lcr} 
Table 1. Characteristics of the 163 enrolled patients. & & \\
\hline Characteristics & Patients N =163 & \\
\hline Mean age (SD), years & $61.6 \pm 9.2$ & 71.17 \\
Gender & 116 & 28.83 \\
Male & 47 & 0.00 \\
Female & & 43.56 \\
Smoking status & 71 & 56.44 \\
Never & 92 & 0.00 \\
Current or ever & 105 & 64.42 \\
ECOG performance status & 58 & 35.58 \\
0-1 & & 0.00 \\
2 & 55 & 33.74 \\
TNM stage & 108 & 66.26 \\
Stage IIIA-IIIB & & 57.06 \\
Stage IV & 93 & 42.94 \\
Histological type & 70 & \\
Non-squamous cell carcinoma & & \\
Squamous cell carcinoma & & \\
\hline
\end{tabular}

\section{Genotypes and response to chemotherapy}

Of the 163 patients, 86 patients showed a CR and PR to chemotherapy (52.76\%) (Table 2). The polymorphic genotypes of rs 11615 and rs3212986 were significantly different between patients who were and were not responsive to chemotherapy treatment. Multivariate regression analysis revealed that individuals carrying the rs 11615 TT genotype and T allele showed a significantly lower response rate to chemotherapy using the rs $11615 \mathrm{CC}$ genotype as a reference $(\mathrm{OR}=0.33,95 \% \mathrm{CI}=0.11-0.91$ for the TT genotype; $\mathrm{OR}=0.53,95 \% \mathrm{CI}=0.33-0.86$ for the $\mathrm{T}$ allele). For rs3212986, carriers of the AA genotype and A allele showed significantly lower response rates to the chemotherapy when compared with the $\mathrm{CC}$ genotype $(\mathrm{OR}=0.17,95 \% \mathrm{CI}$ $=0.038-0.62$ for the AA genotype; $\mathrm{OR}=0.44,95 \% \mathrm{CI}=0.27-0.74$ for the A allele).

\section{Association of genotypes and overall survival}

Until December 2012, 163 patients were followed up, and the median survival time was 19.3 months (range, 2-60 months) (Table 3). A total of 91 patients $(55.83 \%)$ died from NSCLC during the follow-up period. In the Cox proportional hazards model, we found that patients with the rs11615 TT genotype and T allele were significantly associated with an increased risk of death from NSCLC, and the HRs (95\%CIs) for overall survival were 3.74 (1.32-12.63) and 1.97 (1.20-3.34), respectively. Moreover, those carrying the rs3212986 AA 
genotype and A allele also showed an increased risk of death, with HRs (95\%CIs) of 5.41 (1.41-31.73) and 1.99 (1.13-3.35), respectively.

Table 2. Genotypes of 4 ERCC1 SNPs and response to chemotherapy among NSCLC patients.

\begin{tabular}{|c|c|c|c|c|c|c|c|c|c|}
\hline \multirow[t]{2}{*}{ Genotype } & & \multirow[t]{2}{*}{ Cases } & \multirow[t]{2}{*}{$\%$} & \multicolumn{4}{|c|}{ Response to chemotherapy } & \multirow[t]{2}{*}{ OR $(95 \% \mathrm{CI})^{1}$} & \multirow[t]{2}{*}{$\mathrm{P}$ value } \\
\hline & & & & $\mathrm{CR}+\mathrm{PR}$ & $\%$ & $\mathrm{SD}+\mathrm{PD}$ & $\%$ & & \\
\hline \multirow[t]{3}{*}{ rs11615 } & $\mathrm{CC}$ & 76 & 46.63 & 47 & 54.10 & 29 & 37.66 & 1.0 (Ref.) & - \\
\hline & $\mathrm{CT}$ & 61 & 37.42 & 30 & 35.60 & 31 & 40.26 & $0.59(0.29-1.25)$ & 0.14 \\
\hline & TT & 26 & 15.95 & 9 & 10.30 & 17 & 22.08 & $0.33(0.11-0.91)$ & 0.02 \\
\hline \multirow[t]{2}{*}{ Allele } & $\mathrm{C}$ & 213 & 65.34 & 124 & 72.09 & 89 & 57.79 & 1.0 (Ref.) & - \\
\hline & $\mathrm{T}$ & 113 & 34.66 & 48 & 27.91 & 65 & 42.21 & $0.53(0.33-0.86)$ & 0.007 \\
\hline \multirow[t]{3}{*}{ rs3212986 } & $\mathrm{CC}$ & 83 & 50.92 & 52 & 60.40 & 31 & 40.26 & 1.0 (Ref.) & - \\
\hline & $\mathrm{AC}$ & 62 & 38.04 & 30 & 35.20 & 32 & 41.56 & $0.56(0.27-1.15)$ & 0.09 \\
\hline & AA & 18 & 11.04 & 4 & 4.40 & 14 & 18.18 & $0.17(0.038-0.62)$ & 0.002 \\
\hline \multirow[t]{2}{*}{ Allele } & $\mathrm{C}$ & 228 & 70.37 & 134 & 77.91 & 94 & 61.04 & 1.0 (Ref.) & - \\
\hline & A & 98 & 30.06 & 38 & 22.09 & 60 & 38.96 & $0.44(0.27-0.74)$ & $<0.001$ \\
\hline \multirow[t]{3}{*}{ rs2298881 } & AA & 98 & 60.12 & 53 & 61.50 & 45 & 58.44 & 1.0 (Ref.) & - \\
\hline & $\mathrm{AC}$ & 49 & 30.06 & 26 & 30.30 & 23 & 29.87 & $0.96(0.46-2.02)$ & 0.91 \\
\hline & $\mathrm{CC}$ & 16 & 9.82 & 7 & 8.20 & 9 & 11.69 & $0.66(0.19-2.18)$ & 0.44 \\
\hline \multirow[t]{2}{*}{ Allele } & A & 245 & 75.15 & 132 & 76.74 & 113 & 73.38 & 1.0 (Ref.) & - \\
\hline & $\mathrm{C}$ & 81 & 24.85 & 40 & 23.26 & 41 & 26.62 & $0.84(0.49-1.43)$ & 0.48 \\
\hline
\end{tabular}

${ }^{1}$ Adjusted for gender, age, smoking status, and TNM stage. NSCLC $=$ non-small cell lung cancer; $\mathrm{CR}=$ complete response; $\mathrm{PR}=$ partial response; $\mathrm{SD}=$ stable disease; $\mathrm{PD}=$ progressive disease; $\mathrm{OR}=$ odds ratio; $95 \% \mathrm{CI}=95 \%$ confidence interval.

Table 3. Genotypes of 4 ERCC1 SNPs and overall survival among NSCLC patients.

\begin{tabular}{|c|c|c|c|c|c|c|c|c|c|}
\hline \multirow[t]{2}{*}{ Genotype } & & \multirow[t]{2}{*}{ Cases } & \multirow[t]{2}{*}{$\%$} & \multirow[t]{2}{*}{ Death } & \multirow[t]{2}{*}{$\%$} & \multirow[t]{2}{*}{ Alive } & \multirow[t]{2}{*}{$\%$} & Multivariate & \multirow[t]{2}{*}{$P$ value } \\
\hline & & & & & & & & $\mathrm{HR}(95 \% \mathrm{CI})^{1}$ & \\
\hline \multirow[t]{3}{*}{ rs11615 } & $\mathrm{CC}$ & 76 & 46.63 & 36 & 40.1 & 40 & 55.56 & 1.0 (Ref.) & - \\
\hline & $\mathrm{CT}$ & 61 & 37.42 & 35 & 38.2 & 26 & 36.11 & $1.51(0.73-3.26)$ & 0.24 \\
\hline & TT & 26 & 15.95 & 20 & 21.7 & 6 & 8.33 & $3.74(1.32-12.63)$ & 0.006 \\
\hline \multirow[t]{2}{*}{ Allele } & $\mathrm{C}$ & 213 & 65.34 & 107 & 58.79 & 106 & 73.61 & 1.0 (Ref.) & - \\
\hline & $\mathrm{T}$ & 113 & 34.66 & 75 & 41.21 & 38 & 26.39 & $1.97(1.20-3.34)$ & 0.004 \\
\hline \multirow[t]{3}{*}{ rs 3212986} & $\mathrm{CC}$ & 83 & 50.92 & 40 & 43.60 & 43 & 59.72 & 1.0 (Ref.) & - \\
\hline & $\mathrm{AC}$ & 62 & 38.04 & 36 & 39.60 & 26 & 36.11 & $1.50(0.75-3.11)$ & 0.21 \\
\hline & AA & 18 & 11.04 & 15 & 16.80 & 3 & 4.17 & $5.41(1.41-31.73)$ & 0.005 \\
\hline \multirow[t]{2}{*}{ Allele } & $\mathrm{C}$ & 228 & 70.37 & 116 & 63.74 & 112 & 77.78 & 1.0 (Ref.) & - \\
\hline & A & 98 & 30.06 & 66 & 36.26 & 32 & 22.22 & $1.97(1.13-3.35)$ & 0.005 \\
\hline \multirow[t]{3}{*}{ rs2298881 } & $\mathrm{AA}$ & 98 & 60.12 & 53 & 57.70 & 45 & 62.50 & 1.0 (Ref.) & - \\
\hline & $\mathrm{AC}$ & 49 & 30.06 & 29 & 31.60 & 20 & 27.78 & $1.27(0.63-2.73)$ & 0.58 \\
\hline & $\mathrm{CC}$ & 16 & 9.82 & 10 & 10.70 & 6 & 8.33 & $1.46(0.46-5.24)$ & 0.54 \\
\hline \multirow[t]{2}{*}{ Allele } & A & 245 & 75.15 & 135 & 74.18 & 110 & 76.39 & 1.0 (Ref.) & - \\
\hline & $\mathrm{C}$ & 81 & 24.85 & 49 & 26.92 & 32 & 22.22 & $1.29(0.76-2.55)$ & 0.43 \\
\hline
\end{tabular}

${ }^{1}$ Adjusted for gender, age, smoking status, and TNM stage. NSCLC $=$ non-small cell lung cancer; HR $=$ hazard ratio; $95 \% \mathrm{CI}=95 \%$ confidence interval.

\section{DISCUSSION}

In this case-control study, we investigated the prognostic effect of 3 common SNPs in $E R C C 1$ in advanced NSCLC patients receiving platinum-based chemotherapy. Our results showed that the rs11615 and rs3212986 gene polymorphisms were predictive factors for tumor response to chemotherapy and clinical outcome of advanced NSCLC in Chinese patients. Our results are valuable for individualizing treatment for NSCLC patients since it is possible to discriminate patients who are more likely to respond to chemotherapy. 
Previous studies have indicated that several DNA repair genetic polymorphism were correlated with susceptibility to NSCLC, but there have been few studies regarding the association between SNPs in DNA repair genes and clinical outcome of advanced NSCLC. In addition, studies examining SNPs and clinical outcome of NSCLC showed contradictory data. The ERCC1 protein belongs to the nuclear excision repair complex and is involved in the repair of platinum-based induced interstrand and intrastrand cross-links (Mlak et al., 2013). A previous study indicated that the rs 11615 polymorphism is associated with reduced ERCC1 mRNA expression and protein levels, which are associated with reduced repair capacity and survival time (Metzger et al., 2012). More than 100 polymorphisms in the ERCC1 gene have been identified, but few are well understood. Three previous studies indicated that ERCC1 rs11615, rs2298881, and rs3212986 are associated with the prognosis of NSCLC (Takenaka et al., 2010; Mlak et al., 2013; Xu et al., 2013).

In our study, we found that the ERCC1 rs11615 and rs3212986 polymorphisms were associated with NSCLC patients' response to chemotherapy and prognosis. Previous studies indicated that the ERCC1 rs 11615 polymorphism is associated with treatment response in various cancers, including gastric cancer, esophageal cancer, and colorectal cancer (Li et al., 2013; Rumiato et al., 2013; Oguri et al., 2013). One previous study reported that ERCC1 rs11615 is associated with the prognostic markers of clinical outcome of NSCLC (Dong et al., 2012). However, another study conducted in Japan did not observe an association between ERCC1 rs11615 and NSCLC prognosis (Takenaka et al., 2010).

For rs3212986, several previous studies indicated that the ERCC1 rs3212986 polymorphism could be used as a prognostic factor in platinum-based treatment of NSCLC (Takenaka et al., 2010; Mazzoni et al., 2013). Two previous studies conducted in Japan indicated that the ERCC1 rs3212986 CA genotype was significantly associated with poorer disease-free survival and overall survival compared to those with the CC genotype in NSCLC patients treated with platinum-based chemotherapy (Takenaka et al., 2010). Another study conducted in Italy reported that the ERCC1 rs3212986 polymorphism was correlated with increased response to chemotherapy in NSCLC patients. Our results agree with those of previous studies. Studies involving a larger sample size and longer followup period are warranted to confirm the association between SNPs in ERCC1 and clinical outcome of NSCLC.

This study has 2 major limitations. First, because the number of NSCLC patients with variant homozygous genotypes was relatively small, the small sample size limited the statistical power in identifying an association between the ERCCl polymorphisms and clinical outcome of NSCLC. We plan to confirm the findings of our study with a larger sample size in an ongoing study with a longer follow-up time. Second, DNA repair is a complex collection of processes, and thus many DNA repair genes may be involved. In our study, we only examined the role of 3 common functional SNPs in the entire ERCC1 gene. Other functional SNPs in the DNA repair system that may influence the survival of NSCLC should be investigated in a larger study.

In conclusion, we found that polymorphisms in ERCC1 rs11615 and rs3212986 were associated with a poor response to chemotherapy and shorter survival time of advanced NSCLC. The results suggest that the ERCC1 rs11615 and rs3212986 polymorphisms are helpful for identifying patients at high risk of poor clinical outcome, and may contribute to the design of individualized cancer treatment for NSCLC patients. 


\section{ACKNOWLEDGMENTS}

in this study.

We are grateful to the staff of the Fourth People's Hospital of Jinan for their assistance

\section{REFERENCES}

De Dosso S, Zanellato E, Nucifora M, Boldorini R, et al. (2013). ERCC1 predicts outcome in patients with gastric cancer treated with adjuvant cisplatin-based chemotherapy. Cancer Chemother. Pharmacol. 72: 159-165.

Dong J, Hu Z, Shu Y, Pan S, et al. (2012). Potentially functional polymorphisms in DNA repair genes and non-small-cell lung cancer survival: a pathway-based analysis. Mol. Carcinog. 51: 546-552.

Eisenhauer EA, Therasse P, Bogaerts J, Schwartz LH, et al. (2009). New response evaluation criteria in solid tumours: revised RECIST guideline (version 1.1). Eur. J. Cancer 45: 228-247.

Goffin J, Lacchetti C, Ellis PM, Ung YC, et al. (2010). First-line systemic chemotherapy in the treatment of advanced non-small cell lung cancer: a systematic review. J. Thorac. Oncol. 5: 260-274.

IARC - International Agency for Research on Cancer (2012). Lung Cancer. Estimated Incidence, Mortality and Prevalence Worldwide in 2012. Available at [htt://globocan.iarc.fr]. Accessed January 2014.

Krupa R, Synowiec E, Pawlowska E, Morawiec Z, et al. (2009). Polymorphism of the homologous recombination repair genes RAD51 and XRCC3 in breast cancer. Exp. Mol. Pathol. 87: 32-35.

Li Y, Liu Z, Liu H, Wang LE, et al. (2013). ERCC1 and ERCC2 variants predict survival in gastric cancer patients. PLoS One 8: e71994.

Mazzoni F, Cecere FL, Meoni G, Giuliani C, et al. (2013). Phase II trial of customized first line chemotherapy according to ERCC1 and RRM1 SNPs in patients with advanced non-small-cell lung cancer. Lung Cancer 82: 288-293.

Metzger R, Warnecke-Eberz U, Alakus H, Kutting F, et al. (2012). Neoadjuvant radiochemotherapy in adenocarcinoma of the esophagus: ERCC1 gene polymorphisms for prediction of response and prognosis. J. Gastrointest. Surg. 16: 26-34.

Mlak R, Krawczyk P, Ramlau R, Kalinka-Warzocha E, et al. (2013). Predictive value of ERCC1 and RRM1 gene singlenucleotide polymorphisms for first-line platinum- and gemcitabine-based chemotherapy in non-small cell lung cancer patients. Oncol. Rep. 30: 2385-2398.

Molina JR, Yang P, Cassivi SD, Schild SE, et al. (2008). Non-small cell lung cancer: epidemiology, risk factors, treatment, and survivorship. Mayo Clin. Proc. 83: 584-594.

Oguri T, Mitsuma A, Inada-Inoue M, Morita S, et al. (2013). Genetic polymorphisms associated with oxaliplatin-induced peripheral neurotoxicity in Japanese patients with colorectal cancer. Int. J. Clin. Pharmacol. Ther. 51: 475-481.

Ozdemir O, Ozdemir P, Veral A, Uluer H, et al. (2013). ERCC1 expression does not predict survival and treatment response in advanced stage non-small cell lung cancer cases treated with platinum based chemotherapy. Asian Pac. J. Cancer Prev. 14: 4679-4683.

Rumiato E, Cavallin F, Boldrin E, Cagol M, et al. (2013). ERCC1 C8092A (rs3212986) polymorphism as a predictive marker in esophageal cancer patients treated with cisplatin/5-FU-based neoadjuvant therapy. Pharmacogenet. Genomics 23: 597-604.

Siegel R, Naishadham D and Jemal A (2012). Cancer statistics, 2012. CA Cancer J. Clin. 62: 10-29.

Stanczyk M, Sliwinski T, Cuchra M, Zubowska M, et al. (2011). The association of polymorphisms in DNA base excision repair genes XRCC1, OGG1 and MUTYH with the risk of childhood acute lymphoblastic leukemia. Mol. Biol. Rep. 38: 445-451.

Stinchcombe TE and Socinski MA (2008). Considerations for second-line therapy of non-small cell lung cancer. Oncologist 13 (Suppl 1): 28-36.

Tahara E, Yasui W, Ito H and Harris CC (2010). Recent progress in carcinogenesis, progression and therapy of lung cancer: the 19th Hiroshima Cancer Seminar: the 3rd Three Universities' Consortium International Symposium, November 2009. Jpn. J. Clin. Oncol. 40: 702-708.

Takenaka T, Yano T, Kiyohara C, Miura N, et al. (2010). Effects of excision repair cross-complementation group 1 (ERCC1) single nucleotide polymorphisms on the prognosis of non-small cell lung cancer patients. Lung Cancer 67: 101-107.

Wang J, Zhou XQ, Li JY, Cheng JF, et al. (2014). Prognostic significance of ERCC1 expression in postoperative patients with gastric cancer. Chin. J. Cancer Res. 26: 323-330.

Xu TP, Shen H, Liu LX and Shu YQ (2013). Association of ERCC1-C118T and -C8092A polymorphisms with lung cancer risk and survival of advanced-stage non-small cell lung cancer patients receiving platinum-based chemotherapy: A 
pooled analysis based on 39 reports. Gene 526: 265-274.

Yang Z, Fang X, Pei X and Li H (2013). Polymorphisms in the ERCC1 and XPF genes and risk of breast cancer in a Chinese population. Genet. Test. Mol. Biomarkers 17: 700-706. 